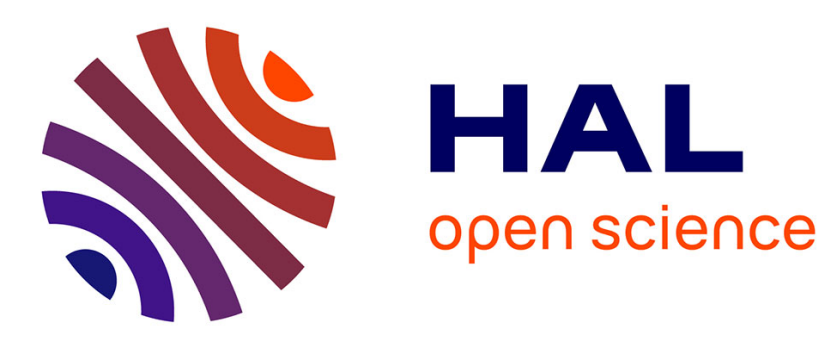

\title{
Estimation of Distributed and Lumped Ohmic Losses in Electrical Cables
}

\author{
Nassif Berrabah, Maud Franchet, Denis Vautrin, Qinghua Zhang
}

\section{To cite this version:}

Nassif Berrabah, Maud Franchet, Denis Vautrin, Qinghua Zhang. Estimation of Distributed and Lumped Ohmic Losses in Electrical Cables. 2016 IEEE International Conference on Antenna Measurements \& Applications, Oct 2016, Syracuse, United States. hal-01376833

\section{HAL Id: hal-01376833 \\ https://inria.hal.science/hal-01376833}

Submitted on 5 Oct 2016

HAL is a multi-disciplinary open access archive for the deposit and dissemination of scientific research documents, whether they are published or not. The documents may come from teaching and research institutions in France or abroad, or from public or private research centers.
L'archive ouverte pluridisciplinaire HAL, est destinée au dépôt et à la diffusion de documents scientifiques de niveau recherche, publiés ou non, émanant des établissements d'enseignement et de recherche français ou étrangers, des laboratoires publics ou privés. 


\section{Estimation of Distributed and Lumped Ohmic Losses in Electrical Cables}

\author{
Nassif Berrabah, Maud Franchet, Denis Vautrin \\ EDF R\&D \\ Ile-de-France, France \\ nassif.berrabah@edf.fr, maud.franchet@edf.fr, denis.vautrin@edf.fr
}

\author{
Qinghua Zhang \\ INRIA \\ Bretagne, France \\ qinghua.zhang@inria.fr
}

\begin{abstract}
Cables play an important role in many industrial systems, from power transmission to data communication. Wired connections are often operated under severe conditions and may be subject to damages or undergo accelerated aging. In order to ensure safe and cost-efficient operations, as well as a high level of performance, efficient tools are needed to assess and monitor the condition of cables. Hard-fault (open or short circuits) are well handled by existing techniques. On the contrary, soft-fault diagnosis still represents an important challenge for current researches. Indeed, their presence in sensor signals is hardly noticeable, while they may be early warning signs of serious failures. This paper focuses on the detection, localization, and estimation of resistive soft fault in electrical cables from reflectometry measurements. A method that enables the computation of the distributed resistance profile $R(z)$ along the cable under test is presented. Both experimental and simulation results are shown as proof of efficiency.
\end{abstract}

\section{INTRODUCTION}

In engineering systems involving wired connections, in order to ensure safety, high performance and cost-effectiveness, it is necessary to monitor the wires for early detection and diagnosis of potential failures. With such issues at stake, the need for efficient condition monitoring and diagnosis tools is strong [1].

In a complex installation, cables travel across several tens of meters and through various environments. Sometimes, cables are operated under severe condition such as extreme temperature, nuclear radiation, humidity, mechanical strain... As a result, they may be subject to accelerated aging and lose some of their properties. Besides, intense localized constraints may result in local faults on the cable. Therefore, the diagnosis of wired connections consists in detection, localization and characterization of both distributed and localized faults.

There exist mechanical and electrical techniques for cable condition monitoring. Among electrical techniques, reflectometry-based methods [2] appear as the most promising method insofar as they permit to account for the non-uniform nature of the tested lines and provide, to some extent, information on the nature of the faults. These techniques may be split into two categories : Time-Domain Reflectometry (TDR) and Frequency-Domain Reflectometry (FDR). Examples of TDR implementation may be found in [3], [4] and those of FDR in [5], [6].

Existing reflectometry-based methods are efficient as to the diagnosis of hard-fault (open or short circuit) but still limited when it comes to soft faults. In the last decade, improvements were brought to standard reflectometry by the introduction of signal processing techniques. Yet, despite these efforts, reflectometry still fails at detecting softest faults [7].

Resistive faults is a kind of soft fault of particular interest as it reveals a damaged conductor and can result in overheating that threatens the cable's isolation integrity. Estimation of the resistance profile $R(z)$ along a cable from reflectometry measurement data constitutes a mean of diagnosis of resistive softfaults. This is an inverse problem that can be solved through non-convex optimization [8], but such a resolution requires a priori information. Moreover, the numerical computations are time-consuming.

In this paper we propose a numerically efficient method for the estimation of $R(z)$ from reflectometry measurements taken at the two ends of a cable. This method is based on ISTL (Inverse Scattering for Transmission Lines), an algorithm capable of estimating the characteristic impedance profile of a cable from reflection coefficient measured at one end. This algorithm was initially designed for lossless cables. However, the results can be influenced by the presence of losses in the probed line. The main idea of the proposed method is to apply ISTL to the two reflection coefficients measured at each end of the cable, and to analyze the difference between the two results in order to estimate $R(z)$. Indeed, if the cable under test was really lossless, then the two estimations of the characteristic impedance profile would be identical. Therefore, the difference between them convey information about the ohmic loss $R(z)$. Results on numerical simulations and experiments will be shown to demonstrate the efficiency of the method.

This paper is organized as follows : In section II a brief recall on transmission lines theory is made and the problem is formulated. Then section III presents the proposed method. Results from simulations and experiments are reported in section IV. Finally a conclusion is drawn in section $\mathrm{V}$

\section{The RlCG Model and Problem Formulation}

For a comprehensive presentation of transmission line theory, including underlying hypothesis and their justification, the reader is refered to [9], [10].

A cable being a transmission line, it is most often represented by the RLCG model as illustrated in Fig. 1. In this 
representation, a cable is seen as a sequence of segments, each of which is represented by a RLCG circuit where:

- $\mathrm{R}$ represents the resistance per unit length of conductors,

- L represents inductance per unit length,

- C represents capacitance per unit length,

- G represents conductance per unit length of insulation.

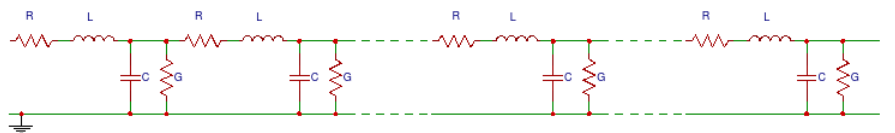

Fig. 1: RLCG Model of a Line

This representation leads to the well-known telegrapher's equations or RLCG model, given in the frequency domain by:

$$
\begin{aligned}
& \frac{\partial u}{\partial z}(z, \omega)=-(R(z)+j \omega L(z)) i(z, \omega) \\
& \frac{\partial i}{\partial z}(z, \omega)=-(G(z)+j \omega C(z)) u(x, \omega)
\end{aligned}
$$

where $\omega$ denotes the pulsation of the propagating sine waves and $u(z, \omega)$ (respectively $i(z, \omega)$ ) denotes the voltage (respectively current) at position $z$ and pulsation $\omega$.

Under these notations, if we connect a network analyzer with internal impedance $Z_{S}$ at the left end $(z=0)$ of the line, then we are able to measure the reflection coefficient:

$$
\Gamma(z=0, \omega)=\frac{Z(z=0, \omega)-Z_{S}}{Z(z=0, \omega)+Z_{S}}
$$

where $Z(z, \omega)$ is the apparent impedance that is defined at any point $z$ along the line as:

$$
Z(z, \omega)=\frac{u(z, \omega)}{i(z, \omega)}
$$

As the parameters $R(z), L(z), C(z)$ and $G(z)$ depends on physical and geometrical properties of the cable at position $z$, their values and variations can be interpreted in order to identify the presence of distributed or localized faults. As a result, the estimation of one or several of them from measurements constitutes a diagnosis method. In what follows we focus on the estimation of the parameter $R(z)$, that is on the diagnosis of resistive soft faults. This will be done using reflectometry measurements under the assumption that $G(z)=0$ (for typical cables, $G(z)$ is at the order of $10^{-9} \mathrm{~S} / \mathrm{m}$ ).

\section{PROPOSED METHOD}

In this section, we detail the steps of the proposed method that performs the estimation of $R(z)$ from Frequency Domain Reflectometry measurements. This method relies on three elements.

1) The fact that under the assumption of low reflection on the line, the characteristic and apparent impedance can be assimilated to each other.

2) The capability to estimate the characteristic impedance of a lossless line from a reflection coefficient.
3) Adding ohmic losses $(R(z))$ to a lossless line modifies the apparent impedance in an easy-to-express manner.

Combining these three items enables to estimate $R(z)$ in a simple and efficient way.

\section{A. ISTL for Lossless Cable Characteristic Impedance Estima- tion}

If the loss parameters $R(z)$ and $G(z)$ are negligible, then the characteristic impedance is defined by the following expression

$$
Z_{0}(z)=\sqrt{\frac{L(z)}{C(z)}} .
$$

It has been suggested in [11] and more recently in [12] that this lossless characteristic impedance can be estimated, by an inverse scattering algorithm, from the reflection coefficient $\Gamma(z=0, \omega)$ measured at one end. This algorithm, implemented in a software named ISTL (Inverse Scattering for Transmission Lines), is numerically efficient [12]. Typically, it takes one or a few seconds for computing the profile $Z_{0}(z)$ at several thousands discretized positions $z$ along the cable.

First, the following variable substitution is introduced:

$$
x(z)=\int_{0}^{z} \sqrt{L(s) C(s)} d s .
$$

$x$ is homogeneous to time and known as the electrical distance along the cable.

Then, the inverse Fourier Transform of the reflection coefficient is computed:

$$
\rho(x)=\operatorname{iFFT}(\Gamma(z=0, \omega)) .
$$

The initial set of equations then amounts to a Gel'fandLevitan-Marchenko set of equations that we solve to get the unknown kernels $A_{1}(x, y)$ and $A_{2}(x, y)$ :

$$
\begin{array}{r}
A_{1}(x, y)+\int_{-y}^{x} A_{2}(x, s) \rho(y+s) d s=0 \\
A_{2}(x, y)+\rho(x+y)+\int_{-y}^{x} A_{1}(x, s) \rho(y+s) d s=0 .
\end{array}
$$

This gives access to a function $q(x)$ that describes the propagation medium and is called scattering potential:

$$
q(x)=2 A_{2}(x, x) .
$$

Finally $Z_{0}(x)$ is extracted from $q(x)$ as:

$$
Z_{0}(x)=Z_{0}(x=0) \exp \left(-2 \int_{0}^{x} q(s) d s\right) .
$$

\section{B. Low reflection under soft fault assumption}

Equation (3) defined the apparent impedance on a cable. We now introduce the characteristic impedance (for a lossy line), which is written:

$$
Z_{c}(z, \omega)=\sqrt{\frac{R(z)+j \omega L(z)}{G(z)+j \omega C(z)}}
$$


The reflection coefficient at any point of the line is expressed as:

$$
\Gamma(z, \omega)=\frac{Z(z, \omega)-Z_{c}(z, \omega)}{Z(z, \omega)+Z_{c}(z, \omega)}
$$

Generally speaking, apparent impedance values differ from characteristic impedance values. However, from (11), it is straightfoward to see that if there are only low reflections on the line $(\Gamma \approx 0)$, then:

$$
Z(z, \omega) \approx Z_{c}(z, \omega)
$$

It is in particular the case if a cable is fault-free or is affected by soft faults (excluding hard faults implying high reflections).

The approximation in (12) holds for all frequencies. In what follows, this relation is used at high frequencies only (mathematically it means the limiting case $\omega \rightarrow \infty$ ), and the notation " $\omega$ " will be dropped for shorter expressions. As $Z_{c}(z, \omega)$ and $Z_{0}(z)$ are equal when $\omega \rightarrow \infty$, in this case the approximation

$$
Z(z) \approx Z_{0}(z)
$$

also holds.

\section{Effect of $R(z)$ on $Z(z)$}

The apparent impedance $Z(z)$ depends on the boundary conditions at the two ends of a cable. Let us consider two particular cases. In the first case a network analyzer is connected to the left end and a matched load is connected to the right end. The apparent impedance in this case is denoted $Z^{(l)}(z)$. In the second case, the positions of the network analyzer and the load are inverted, and the corresponding apparent impedance is denoted by $Z^{(r)}(z)$.

For the time being, assume the cable under test is lossless. Consider $Z^{(l)}(z)$ at two neighbor positions $z$ and $z+\delta z$ and let $\delta Z(z)$ denote their difference, then:

$$
Z^{(l)}(z+\delta z)=Z^{(l)}(z)+\delta Z(z) .
$$

Now insert a resistance $R(z)$ between positions $z$ and $z+\delta z$, then

$$
Z^{(l)}(z+\delta z)=Z^{(l)}(z)+\delta Z(z)-R(z) \delta z
$$

If $R(z)$ is inserted at every segment of length $\delta z$, or equivalently, if $R(z)$ accounts for distributed resistive losses, then the resulting cable represented in Fig. 2 is equivalent to that of Fig. 1 in the case $G=0$. Similarly,

$$
Z^{(r)}(z+\delta z)=Z^{(r)}(z)+\delta Z(z)+R(z) \delta z
$$

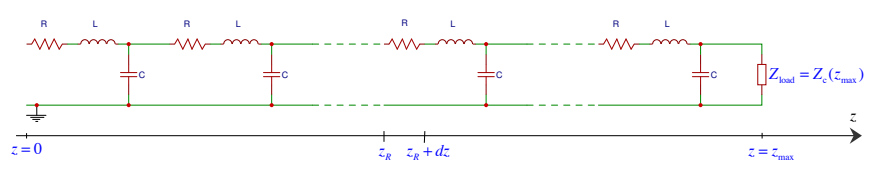

Fig. 2: Effect of $\mathrm{R}(\mathrm{z})$ on apparent impedance

\section{Retrieving $R(z)$}

The following three-steps procedure is applied to estimate $R(z)$ :

1) From each of the two reflection coefficients measured at the two ends of a cable, we calculate two estimations $Z_{0}^{(l)}(z)$ and $Z_{0}^{(r)}(z)$ of its characteristic impedance, using ISTL as if the cable was lossless (if it actually was, the two results would be identical).

2) Under the low-reflection (soft-fault only) assumption, $Z_{0}^{(l)}(z)$ and $Z_{0}^{(r)}(z)$ are approximately equal to the apparent impedances in the corresponding configurations:

$$
Z_{0}^{(l)}(z) \approx Z^{(l)}(z) \quad \text { and } \quad Z_{0}^{(r)}(z) \approx Z^{(r)}(z)
$$

3) We combine 15 and 16 to get the estimation of $R(z)$ :

$$
R(z) \approx \frac{\left[Z^{(l)}(z)-Z^{(r)}(z)\right]-\left[Z^{(l)}(z+\delta z)-Z^{(r)}(z+\delta z)\right]}{2 \delta z}
$$

\section{RESULTS}

In order to provide evidence of the effectiveness of our method, we applied it to two practical cases, both experimental and numerical. The former is performed to prove that the method works for the diagnosis of localized ohmic loss faults and the latter is used for testing the method in the case of distributed losses.

\section{A. Laboratory Results for Localized Faults}

We present here the results from two lab experiments that involve localized faults on a cable.

In a first experiment, we performed measurement for frequencies from DC to $f_{\max }=5 \mathrm{GHz}$ with a frequency step of $d f=1 \mathrm{MHz}$ on a $40 \mathrm{~m}$-long coaxial cable. In the middle position, a $49.7 \Omega$ (measured with Fluke 8845A) resistor (standard component for electronic circuits) has been inserted in the cable core. This inserted resistor imitates a localized resistive fault. An outline of the setup and pictures are shown in Fig. 3

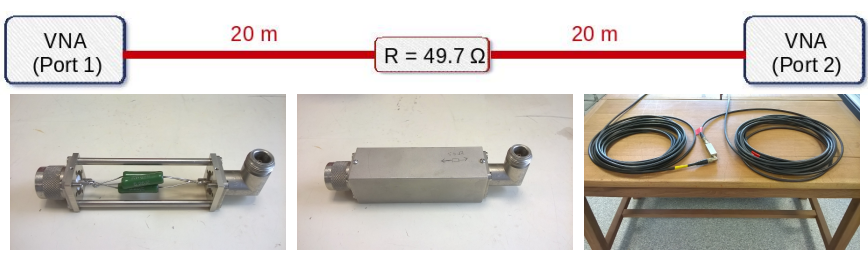

Fig. 3: Case 1: $40 m$-long cable with a $49.7 \Omega$ resistive fault

The obtained result is shown on Fig. 4 The resistance per unit length $R(z)$ is estimated and a peak appears at the expected position of the fault. The ordinate of Fig. 4 is the resistance per unit length $(\Omega / m)$, hence the height of the spike of $R(z)$ does not directly indicate the lumped resistance of $49.7 \Omega$ inserted at the position of the peak. In principle a lumped resistance corresponds to a Dirac function spike in $R(z)$. In practice due to limited bandwidth of the spectral measurements, the spike of $R(z)$ has a finite height and spreads out slightly. In Fig. 4 a zoom around the spike 
confirms this fact. Nevertheless, the integral of $R(z)$ around the spike provides an estimation of the lumped resistance. In this example the integral value is $47.15 \Omega$. Compared to the actual resistance value of $49.7 \Omega$, the estimation error is within $5 \%$ of the resistance.

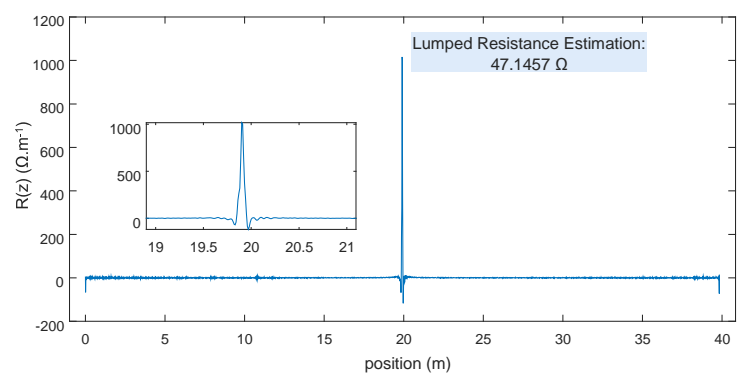

Fig. 4: Results obtained for case 1

A second experiment run on a similar setup is shown in Fig. 5. The frequencies of measurements are the same. This time the inserted resistor has a resistance value of $18.3 \Omega$ and is placed at approximately $20 \mathrm{~m}$ from one end on a $25 \mathrm{~m}$-long cable.
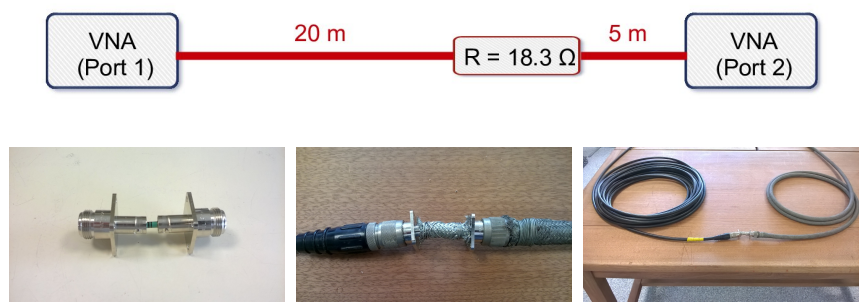

Fig. 5: Case 2: $25 m$-long cable with a $18.3 \Omega$ resistive fault

The result obtained is shown on Fig. 6. The parameter profile $R(z)$ is again estimated with good accuracy as the fault is localized at the expected position and the lumped resistance value is assessed as $17.96 \Omega$ (estimation error within $2 \%$ ).

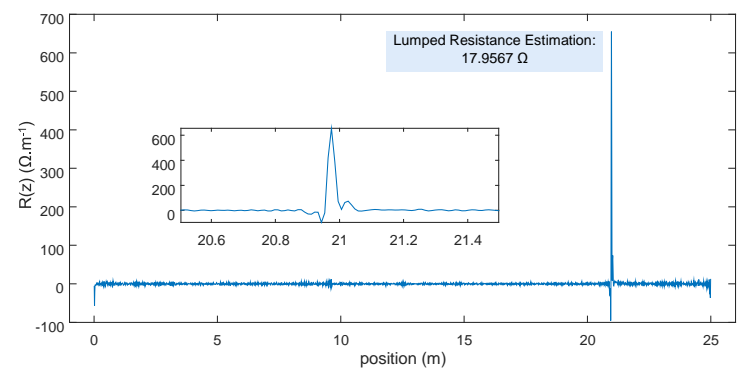

Fig. 6: Results obtained for case 2

\section{B. Simulation Results for Distributed Faults}

It is more difficult to create distributed faults in a controlled manner in laboratory experiments. We ran simulations to demonstrate the performance of the method in estimating a continuously varying smooth resistance profile $R(z)$. The result is displayed in Fig. 7. The blue line shows the simulated resistance profile used to generate data whereas the resistance estimated from this data is represented by the black dashed line. The estimation fits almost perfectly the simulated curve.

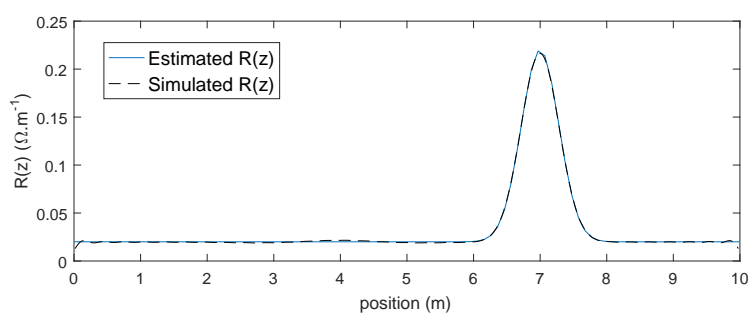

Fig. 7: Simuation results for distributed resistance

\section{CONClusion}

Diagnosis of soft-faults in cables is of crucial importance in many sectors. We proposed a method that estimates the profile of resistance per unit length all along a cable from measurements at both ends, thus providing detection, localization and estimation of resistive ohmic faults. These faults can be due to a damaged conductor and/or produce hazardous overheating. The proposed method needs no a priori information on $R(z)$, and can be executed with fast computations. Moreover, it allows to distinguish faults affecting the resistance from faults affecting the impedance. It can be used for diagnosis as well as condition monitoring.

\section{REFERENCES}

[1] C. Furse, "Down to the Wire," IEEE Spectrum, pp. 34-39, 2001.

[2] F. Auzanneau, "Wire Troubleshooting and Diagnosis - Review and Perspectives," Progress In Electromagnetics Research, vol. 49, no. February, pp. 253-279, 2013.

[3] M. K. Smail, "Développement d'une méthodologie dédiée à la réflectométrie en vue du diagnostic filaire," Ph.D. dissertation, Université Paris-Sud XI, 2010.

[4] L. El Sahmarany, L. Berry, N. Ravot, F. Auzanneau, and P. Bonnet, "Time Reversal for Soft Fault Diagnosis in Wire Networks," PIER M, vol. 31, no. May, pp. 45-58, 2013.

[5] Y. Ohki and N. Hirai, "Effects of the Structure and Insulation Material of a Cable on the Ability of a Location Method by FDR," IEEE Transaction on Dielectric and Electrical Insulation, vol. 23, no. 1, 2016.

[6] C. Furse, Y. C. Chung, R. Dangol, M. Nielsen, G. Mabey, and R. Woodward, "Frequency-domain reflectometery for on-board testing of aging aircraft wiring," IEEE Transactions on Electromagnetic Compatibility, vol. 45, no. 2, pp. 306-315, 2003.

[7] L. A. Griffiths, R. Parakh, C. Furse, and B. Baker, "The invisible fray: a critical analysis of the use of reflectometry for fray location." 2006.

[8] M. Norgren and S. He, "An optimization approach to the frequencydomain inverse problem for a nonuniform LCRG transmission line," IEEE Transactions On Microwave Theory And Techniques, vol. 44, no. 8 , pp. 1503-1507, 1996.

[9] C. R. Paul, Analysis of multiconductor transmission lines, 2nd ed. Hoboken, New Jersey: John Wiley \& Sons, 2008.

[10] R. E. Collin, Field theory of guided waves, 2nd ed. IEEE Press, 1991.

[11] M. Jaulent, "The inverse scattering problem for L C R G transmission lines," Journal of mathematical physics, pp. 2286-2290, 1982.

[12] Q. Zhang, M. Sorine, and M. Admane, "Inverse scattering for soft fault diagnosis in electric transmission lines," IEEE Transactions on Antennas and Propagation, vol. 59, no. 1, pp. 141-148, 2011. 